Discussion Paper No. 814

\title{
OVERCONFIDENCE INCREASES \\ PRODUCTIVITY
}

\author{
Yusuke Kinari \\ Noriko Mizutani \\ Fumio Ohtake \\ Hiroko Okudaira
}

August 2011

The Institute of Social and Economic Research

Osaka University

6-1 Mihogaoka, Ibaraki, Osaka 567-0047, Japan 


\title{
Overconfidence Increases Productivity*
}

\author{
Yusuke Kinari $^{\mathrm{a}}$ \\ Graduate School of Economics, Kyushu University
}

Noriko Mizutani ${ }^{\mathrm{b}}$

Institute for Research on Household Economics

Fumio Ohtake ${ }^{\mathrm{c}}$

Institute of Social and Economic Research, Osaka University

Hiroko Okudaira $^{\mathrm{d}}$

Graduate School of Economics, Okayama University

\footnotetext{
* We thank Kan Takeuchi, Hideaki Kato, and participants at the fourth annual meeting of the Association of Behavioral Economics and Finance. This work was financially supported by a Grant-in-Aid for Challenging Exploratory Research from the Japan Society for the Promotion of Science and the Collaborative Program of the Institute of Social and Economic Research, Osaka University.

${ }^{\text {a }}$ Corresponding author: Graduate School of Economics, Kyushu University, 6-19-1, Hakozaki, Higashi-ku, Fukuoka, 812-8581, Japan. Tel: +81-92-642-2493; e-mail: kinari@en.kyushu-u.ac.jp.

${ }^{\mathrm{b}}$ Institute for Research on Household Economics, 2-3-7, Kudan-kita, Chiyoda-ku, Tokyo, 102-0073, Japan. E-mail: mizutani@kakeiken.or.jp.

${ }^{c}$ Institute of Social and Economic Research, Osaka University, 6-1, Mihogaoka, Ibaraki, 567-0047, Japan. E-mail: ohtake@iser.osaka-u.ac.jp.

d Graduate School of Economics, Okayama University, 3-1-1, Tsushima-naka, Kita-ku, Okayama 700-8530, Japan.E-mail: okudaira@e.okayama-u.ac.jp.
} 


\title{
Overconfidence Increases Productivity
}

\author{
Abstract \\ Recent studies report that productivity increases under tournament reward structures \\ than under piece rate reward structures. We conduct maze-solving experiments under \\ both reward structures and reveal that overconfidence is a significant factor in \\ increasing productivity. Specifically, subjects exhibiting progressively higher degrees of \\ overconfidence solve more mazes. This result shows a positive aspect of overconfidence, \\ which usually has been examined in its negative aspect as an expectation bias.
}

JEL: C9, D03, J33

Keywords: Expectation, Overconfidence, Reward structure, Productivity, Experiment 


\section{Introduction}

There are many ways of paying rewards. Under a piece rate reward structure, the reward people earn depends solely on their individual productivity, whereas under a tournament reward structure, it depends on not only their productivity but also their competitors' productivities. It is important to know how different reward structures affect productivity. In the field of labor economics and business, such knowledge could guide firms in setting employee rewards to increase profits, and in the context of education, it helps educators understand how to motivate children toward their best efforts.

Recent studies examine the relationship between reward structures and productivity and report that productivity increases under the tournament structure compared with the piece rate structure (Gneezy et al., 2003; Freeman and Gelber, 2010; Mizutani et al., 2009; Niederle and Vesterlund, 2007). ${ }^{1}$ Freeman and Gelber (2010), whose research is most relevant for this study, conducted maze-solving experiments and investigated the effects of the tournament prize structure and the disclosure of information about other subjects' skills. They asked groups of six subjects to participate in two rounds of maze-solving problems. In Round 1, subjects were rewarded by piece rate. After the groups completed Round 1, researchers informed subjects in half of the groups about the distribution of mazes solved in Round 1 in their groups, but they withheld that information from subjects in the remaining half. In Round 2, subjects solved mazes under three different reward structures: fixed, single-prize tournament, and multiple-prize tournament. Using the number of mazes solved as the measure of

\footnotetext{
${ }^{1}$ Although Mizutani et al. (2009) and Niederle and Vesterlund (2007) do not focus on productivity, their experimental results demonstrate that productivity increases under the tournament structure compared with the piece rate structure.
} 
performance, they found that performance was lowest under the fixed-reward structure, higher under the single-prize tournament structure, and highest under the multiple-prize tournament structure. They also found that revealing information on performance of other group members affected productivity. When this information was disclosed to subjects who scored highly in Round 1, their subsequent performance was greater than when the information was withheld. In contrast, performance among subjects who scored low in Round 1, decreased when the information was disclosed and increased when it was withheld.

Freeman and Gelber (2010) interpret their results as demonstrating the effect of incentives on effort. Under the multiple-prize tournament structure, all subjects have an incentive to increase their rewards, whereas the fixed-prize structure provides no such incentive. Under the single-prize structure, subjects with a high chance of winning earn the incentive, and those with a low chance of winning lose the incentive.

This study examines the changes in productivity that occur when subjects receive no information about the distribution of competitors' skills. We conduct maze-solving experiments under the piece rate and tournament reward structures and explore the effects of subjective expectations and overconfidence on productivity. Subjects are divided into groups of four individuals and are asked to anticipate their rankings within their groups. If we offer information about other subjects' skills, it would act as an incentive; if we withhold information about other subjects' skills, subjective expectations would act as the incentive. That is, if subjects regard themselves as good performers, they will try harder to win the tournament. If they do not regard themselves as good performers, they will give up the attempt to win the tournament.

These subjective expectations are based on two factors: subjects' actual ability and 
their confidence levels. We refer to the latter as "overconfidence” or "underconfidence.” The results of Freeman and Gelber (2010) suggest that overconfidence may affect productivity. They report that the performance of subjects who scored low in Round 1 increases when the information regarding other subjects' performance is withheld. This may occur because subjects do not realize that their skills are less than their competitors' - that is, they are overconfident. Moreover, it is considered that the performance of subjects who scored highly in Round 1 decreases more when the information is withheld because they are underconfident.

We have other grounds for concluding that overconfidence increases productivity. Gneezy et al. (2003) conduct maze-solving experiments and observe a gender difference in increases in performance from the piece rate to the tournament reward structure. In particular, female subjects perform better in all-female groups, whereas their performance does not increase in mixed-gender groups. Mizutani et al. (2009) conduct computational experiments and report that women become overconfident in all-female groups, whereas they are under-confident in mixed-gender groups. These results imply that overconfidence increases productivity and that a gender difference in overconfidence causes the gender difference in increased productivity. We test this hypothesis using actual overconfidence measures constructed from participants' subjective estimates of their rankings within their groups and the number of mazes solved.

The remainder of this paper is organized as follows. Section 2 describes our experiment. Section 3 presents our results and reveals that subjective expectations and overconfidence affect subjects’ performance significantly. Specifically, subjects who expect worse rankings increase their performance more than those who expect better 
rankings, and overconfident subjects increase their performance. This is evidence that overconfidence has a positive aspect, which previously has been studied mainly in its negative aspect as an expectation bias. Section 4 concludes.

\section{Method}

We conducted the experiment at Osaka University on October 25, November 15 and 29, and December 6, 2009. Subjects included 132 students (67 males and 65 females) affiliated with Osaka University. We assigned subjects to groups of four participants and asked them to solve mazes. Subjects were not aware of which members were included in their groups, but knew their groups' gender ratios. We organized groups according to five gender ratios: nine groups of four males; one group of three males and one female; 13 groups of two males and two females; two groups of one male and three females; and eight groups of four females each. ${ }^{2}$ Our subjects' tasks included solving as many mazes as possible within three minutes, and the time remaining always appeared with the maze on their computer screens. Subjects moved cursors from the start to the goal point using arrow keys on their keyboards. They could skip a maze by clicking a radio button on their screens and move to the next maze whenever they were willing to do so. The number of skips was unlimited.

The experiment consisted of three tasks-Task 1, Task 2, and Task 3. For Task 1, we rewarded subjects by piece rate; their reward of $¥ 100$ per maze solved depended solely on their individual performance. Task 2 was completed under the tournament reward structure; only the winner in each group received a reward of $¥ 400$ per maze solved. For

\footnotetext{
${ }^{2}$ We initially intended to conduct our experiments using three gender groups (all-male, all-female, and two males-two females). However, two additional groups (one male-three females and three males-one female) emerged as we recruited subjects hastily to replace those who cancelled at the last minute.
} 
Task 3, subjects could choose the piece rate or tournament reward structure before solving mazes, and rewards were paid on the basis of their choices. To accustom subjects to maze-solving using a computer, we conducted one-minute training sessions before Task 1.

In addition, subjects were asked to anticipate their ranking within their groups after completing each task. We asked them not only their expected rankings but also their probability distribution forecasts. In estimating the expected ranking, subjects pick a rank between 1 and 4. In estimating the probability distribution, subjects were required to give their expected probabilities of each rank by percentages. We also asked them to complete a questionnaire at the end of the experiment to measure their attitudes toward risk and competition.

All subjects received a ¥3,000 show-up fee for participation. At the end of the experiment, we randomly selected one task among the three, for which we actually paid the reward. Subjects were paid on the basis of their performance in the tasks plus the show-up fee.

\section{Results}

\subsection{Gender differences in performance}

Table 1 reports the mean number of mazes solved and the standard errors for Tasks 1 and 2. For both tasks, the mean number of mazes solved by males was greater than that by females. The gender differences in mean mazes solved are approximately 2.5 in Task 1 and 1.9 in Task 2 and are significant at 1\%. Niederle and Vesturland (2007) and Mizutani et al. (2009) conduct computational experiments under the piece rate and tournament structures and find no gender differences in performance, perhaps indicating 
gender differences in the ability to solve mazes. ${ }^{3}$

Table 2 presents the change in performance from Task 1 to Task 2. Both males and females increase their performance significantly. For male subjects, the mean performance increases about 0.9 (9.4\%), and for female subjects, it increases about 1.5 (18.5\%). The gender difference in increase in performance, approximately $0.6(9.1 \%)$, is significant at $1 \%$. Our result is inconsistent with that of Gneezy et al. (2003), who report that performance of males improve under the tournament compared with that under the piece rate, but female performance do not. However, this result does not reject our hypothesis that the gender difference in overconfidence causes the gender difference in increased performance. This is because females were more overconfident than males in our experiment, as reported in 3-3.

\subsection{Expected ranking and performance}

Table 3 demonstrates subjects' performance in Tasks 1 and 2 and compares the change in performance with subjects' expected rankings following Task 1 . While nearly half the subjects estimated that they ranked second within their groups, only three subjects believed they ranked fourth. Subjects' actual performance tends to be lower when their estimated ranking is lower, indicating that subjects to some extent ranked themselves reasonably. However, given that more than half estimated their rankings as above average, the result resembles that of Svenson (1981), who reports that a majority of subjects regarded themselves as more skillful and less risky than the average driver.

On the other hand, performance in Task 2 tends to increase when subjects' expected rankings following completion of Task 1 were lower. That is, the increase in

\footnotetext{
3 Perhaps gender stereotyping produces the result. In fact, Steele and Ambady (2006) and Shih, Pittinsky, and Ambady (1999) report that gender stereotypes influenced performance.
} 
performance of subjects who ranked themselves lower was greater than that of subjects who ranked themselves higher. This result differs slightly from Freeman and Gelber (2010), who report that the increase in performance among highly-skilled subjects is greater than the increase among lowly-skilled subjects, even when subjects have no information about other subjects' skills. Our result may indicate that individuals never give up trying to win, even when they ranked themselves low.

\subsection{Overconfidence and performance}

We asked subjects to estimate their rankings within their groups after each task. This expected ranking is based on two factors: subject's actual ability and their confidence level. We refer to the latter as “overconfidence” or "underconfidence.” Many studies document gender differences in overconfidence. ${ }^{4}$

We define overconfidence numerically by deducting expected ranking from subject's actual ranking after completing both the tasks. As a proxy for each subject's actual ranking, we use his or her average ranking within the group generated by 1 million times random grouping. As a proxy for each subject's expected ranking, we use both the mean of the expected probability distribution (Overconfidence 1) and the point forecast (Overconfidence 2). For both measures, a positive (negative) value implies that subjects are overconfident (underconfident) because their expected rankings were higher (lower) compared with their actual ranking.

Table 4 shows the means of the overconfidence measures and their gender differences. Means of overconfidence measures for all subjects are 0.266 for Overconfidence 1 and 0.391 for Overconfidence 2. Both are significant at $1 \%$, which implies that our subjects

\footnotetext{
${ }^{4}$ Croson and Gneezy (2009) introduced many studies on the gender difference in overconfidence.
} 
are generally overconfident. Furthermore, women are more overconfident than men in both measures. Gender differences are significant at 1\% for Overconfidence 1 and at 10\% for Overconfidence 2. Previous literature reports that men are more overconfident than women (Barber and Odean, 2001). Mizutani et al. (2009) conduct almost the same experiment employing computational rather than maze-solving tasks, and they report that male subjects are more overconfident than female subjects. These results indicate that gender differences in overconfidence depend on the task assigned.

Figure 1 shows the means of Overconfidence 1 by the change in performance from Task 1 to Task 2. The horizontal and vertical axes present the change in performance from Task 1 to Task 2 and the degree of overconfidence, respectively. We find a positive correlation between the increase in performance and degree of overconfidence. Subjects whose performance increased are generally overconfident, as we define it here, and those whose performance decreased are generally underconfident. Further, greater increases in performance are associated with greater degrees of overconfidence. It is noteworthy that subjects whose performance does not change are neither overconfident nor underconfident.

Overconfidence has been studied mainly in its negative aspect as an expectation bias. Barber and Odean (2001) report that overconfidence increases frequency of stock trading, but returns decrease because of high transaction costs. Mizutani et al. (2009) and Niederle and Vesturland (2007) report that overconfidence leads subjects to overestimate their capabilities and enter competitions, which consequently decreases their returns. While these studies emphasize how overconfidence decreases returns, our experiment demonstrates that overconfidence can improve subjects' performance, even if their performance does not improve sufficiently to earn or increase a reward. This is 
evidence that overconfidence has a positive aspect. ${ }^{5}$

\subsection{Gender and performance}

Table 5 presents the change in performance from Task 1 to Task 2 by gender and gender grouping, although one group of three males-one female and two groups of one male-three females are excluded because the number of observation is few. Male subjects in two male-two female groups improve their performance compared with those in all-male groups, although the difference is not significant. The gender composition of groups did not affect female subjects' performance. Women improve their performance from Task 1 to Task 2 irrespective of whether men belong to their group. This finding is inconsistent with that of Gneezy et al. (2003), suggesting that the effect of gender on performance may differ by country or culture.

Table 6 shows the means of overconfidence measures by gender and gender grouping, although one group of three males-one female and two groups of one male-three females are excluded because the number of observations is few. Male subjects tend to be more overconfident when grouped with females, and female subjects tend to be more overconfident in all-female groups. These results are consistent with those of Mizutani et al. (2009), although differences in overconfidence measures between groups are insignificant.

Results from Tables 5 and 6 are inconsistent with those of Gneezy et al. (2003) and Mizutani et al. (2009) in that differences in the changes in performance and overconfidence between groups are not significant. However, these results still support

\footnotetext{
${ }^{5}$ Several recent studies demonstrate the positive roles of overconfidence. Galasso and Simcoe (2010) and Hirshleifer et al. (2010) reveal that CEO overconfidence encourages innovation. The latter also find that CEO overconfidence increases the firm's profitability. Anderson and Brion (2010) reveal that overconfidence helps individuals gain status.
} 
our hypothesis that overconfidence increases performance. Tables 5 and 6 demonstrate that overconfidence and performance tend to correlate positively, although the correlation for women is ambiguous.

\subsection{Regression analysis}

We run the OLS regression to evaluate quantitatively the effect of expected rankings and overconfidence on increase in performance. We use the increase in performance and the rate of increase as dependent variables. As independent variables, we use dummy variables constructed by subjects' point forecasts for Task 1 and Overconfidence 1 plus a female dummy and a constant term. ${ }^{6}$

Table 7 presents the regression results. In Eq.1, the coefficient of the constant term is significantly positive at $1 \%$, indicating that productivity increases under the tournament reward structure. This result is consistent with previous literature. The coefficient of the female dummy is also significantly positive at $1 \%$, which is inconsistent with the result of Gneezy et al. (2003). However, as noted above, this result does not reject our hypothesis that the gender difference in overconfidence causes the gender difference in increased performance, for women are more overconfident than men in our experiment.

In Eq.2, we added Overconfidence 1 as an independent variable in Eq.1. The coefficient of Overconfidence 1 is significantly positive at $1 \%$, but the coefficient of the female dummy is no longer significant. These results support our hypothesis that the gender difference in overconfidence causes gender difference in the increase in performance.

\footnotetext{
${ }^{6}$ We use expected rankings on Task 1 rather than Task 2 because we wish to investigate how subjects' expectations affect subsequent performance. To use expected rankings of Task 1 is useful in that we can ignore the endogeneity problem that subjects ranked themselves low because they did not perform well.
} 
In Eq.3, we added dummies for subjects’ expected rankings as independent variables to Eq.1. Coefficients of all dummies are positive, and dummies for second- and fourth-place rankings are significant at $10 \%$. This result indicates that subjects who ranked themselves second and fourth improved their performance more than subjects who ranked themselves first. The significance of the constant term weakened compared with Eq.1, indicating that performance increases under the tournament reward structure are engendered by the incentive to earn higher rankings or greater rewards. Our results are consistent with that of Freeman and Gelber (2010) in suggesting that subjects try harder to win and elevate their performance. However, they differ from Freeman and Gelber (2010) in that the increase in performance of subjects who ranked themselves lower within their groups exceeded that of subjects who ranked themselves higher.

In Eq.4, we added Overconfidence 1 and the expected ranking dummies to Eq.1. Coefficients of the constant term and the female dummy are not significant, whereas coefficients are significantly positive for Overconfidence 1 and the expected ranking dummies. Therefore, we retain our result that the increase in performance and its gender difference are brought about by incentives for higher rankings or larger rewards and overconfidence.

Table 8 shows the results of OLS regressions using the rate of increase in performance from Task 1 to Task 2 as the dependent variable. The results robustly support our hypothesis. Coefficients of the expected ranking dummies and Overconfidence 1 are significantly positive. Subjects who ranked themselves lower tend to increase performance more than subjects who ranked themselves higher. Furthermore, the significance of the constant term and the female dummy are weakened by adding Overconfidence 1 and the expected ranking dummies to the regression. Our results 
remained unchanged when we added the variance of the expected probability distribution, risk aversion, the number of skips in solving mazes, dummy variables for gender ratio, and attitudes toward competition. Our results are also retained when we substituted Overconfidence 2 for Overconfidence 1 as an independent variable.

\subsection{Learning and reward structure}

To accustom themselves to maze-solving on a computer, subjects attended one-minute training sessions before Task 1 . However, we cannot rule out the possibility that one minute was too brief for subjects to master their tasks and that the increase in performance from Task 1 to Task 2 indicates a learning effect.

To measure the effects of overconfidence and expected rankings while controlling for the learning effect, we run the OLS regressions after restricting the sample to subjects who chose the tournament reward structure for Task 3-the same reward structure as Task 2. Thus, we can control for both the learning effect and attitudes toward competition. Dependent variables are the increase in performance from Task 2 to Task 3 and its rate of increase. Independent variables are the dummies constructed by subjects' point forecasts in Task 2. We also use Overconfidence 1 as an independent variable, which is calculated on the basis of subjects' performance and their expected rankings for Task 2.

Table 9 presents the results of OLS regression. In Eqs. 1 and 3, the constant terms are not significant, indicating we can dismiss the learning effect and the effect of the reward structure. On the other hand, we find the gender difference in increase in performance even from tournament to tournament. Although insignificant in Eq.1, the female dummy is significant at $10 \%$ in Eq.3. However, its significance weakens when we add 
Overconfidence 1 and the expected ranking dummies as independent variables, supporting our hypothesis that the gender difference in overconfidence causes the gender difference in increased performance. Overconfidence remains significant for increased performance: its coefficient is significantly positive at $1 \%$. Conversely, it is unclear whether performance of subjects, who ranked themselves lower, increases more than performance of subjects who ranked themselves higher. The coefficient of the second-rank dummy is significant at $5 \%$, but that of the third-rank dummy is insignificant. ${ }^{7}$ However, subjects who expected their rankings second within their groups increased their performance more than those who expected their rankings first. Accordingly, our results are robust with regard to the learning effect and subjects' attitudes toward competition.

\subsection{Upper half and lower half}

Freeman and Gelber (2010) report that subjects who ranked poorly in ability do not increase their performance, whereas those who ranked highly increase their performance when informed about the distribution of mazes solved in their group. Based on our finding that overconfidence increases performance, we could re-interpret the result of Freeman and Gelber (2010). We might conclude that overconfidence among lesser-performing subjects is corrected by providing them objective information and as a result their performance does not increase. If this is the case, lower-performing subjects might increase their performance when information about the distribution of mazes solved is withheld.

Table 10 presents the results of OLS regressions by subjects' skills. We divided the

\footnotetext{
${ }^{7}$ No subjects in the restricted sample expected their rankings to be the lowest.
} 
sample into upper and lower halves based on their average rankings in a group of four subjects generated by 1 million times random grouping. Subjects in the lower half who ranked themselves fourth in their groups increased their performance, implying that we could improve their performance by withholding the objective information — that is, by not correcting overconfidence. It is noteworthy that the effect of overconfidence for the upper half is stronger than for the bottom half. It may imply that the effect of overconfidence on increased performance depends on subjects' skill.

\section{Conclusion}

This study has clarified the effect of subjective expectations and overconfidence on productivity by conducting experiments in which subjects solved as many mazes as possible in three minutes. Subjects were divided into groups of four. In Task 1, subjects solved mazes under a piece rate reward structure that paid $¥ 100$ per correct answer. In Task 2, they solved mazes under a tournament reward structure in which only the winner received $¥ 400$ per correct answer. In Task 3, subjects could choose either reward structure and were paid based on their choices. They were asked to anticipate their rankings within their groups after completing each task.

Previous literature reveals that performance increases under the tournament reward structure. Freeman and Gelber (2010), the research most relevant for this study, conclude that incentives contribute substantially to improving performance, and they investigate how performance changes by informing subjects' about the performance of other group members. However, we focused on subjective expectations, which serve as an incentive when information about other subjects' performance is withheld. In addition, Gneezy et al. (2003) report a gender difference in increased performance under 
the tournament reward structure. We focus on the gender difference in overconfidence as a cause of the gender difference in higher performance, and examine the effect of overconfidence on productivity.

We found that subjective expectations and overconfidence significantly affect productivity. Subjects who ranked themselves lower than other group members increase their performance more than subjects who ranked themselves higher, and overconfident subjects increase their performance. This reveals the positive aspect of overconfidence, which previously has been examined mainly in its negative aspect as an expectation bias. 
References

Anderson, Cameron and Sebastien Brion (2010), “Overconfidence and the attainment of status in groups,” Working Paper Series, Institute for Research on Labor and Employment, UC Berkeley.

Barber, Brad M. and Terrance Odean (2001), "Boys will be Boys: Gender, Overconfidence, and Common Stock Investment,” Quarterly Journal of Economics, 116(1), pp. 261-292.

Croson, Rachel and Uri Gneezy (2009), “Gender Differences in Preferences,” Journal of Economic Literature, 47(2), pp.448-474.

Freeman, Richard B. and Alexander M. Gelber (2010), "Prize Structure and Information in Tournaments: Experimental Evidence,” American Economic Journal: Applied Economics, 2(1), pp.149-164.

Galasso, Alberto and Timothy S. Simcoe (2010), “CEO Overconfidence and Innovation,” NBER Working paper No. 16041.

Gneezy, Uri, Muriel Niederle, and Aldo Rustichini (2003), "Performance in Competitive Environments: Gender Differences,” Quarterly Journal of Economics, 118(3), pp. 1049-1074. 
Hirshleifer, David A., Angie Low, and Siew H. Teoh (2010), “Are Overconfident CEOs Better Innovators?” MPRA Paper No. 22425.

Mizutani, Noriko, Hiroko Okudaira, Yusuke Kinari, and Fumio Ohtake (2009), “Overconfidence Makes Men Compete More,” Journal of Behavioral Economics and Finance, 2(1), pp.1-26, (in Japanese).

Niederle, Muriel and Lise Vesturland (2007), "Do Women Shy Away from Competition? Do Men Compete Too Much?,” Quarterly Journal of Economics, 122(3), pp.1067-1101.

Shih, Margaret, Todd L. Pittinsky, and Nalini Ambady (1999), "Stereotype Susceptibility: Identity Salience and Shifts in Quantitative Performance,” Psychological Science, 10(1), pp.80-83.

Steele, Jennifer R. and Nalini Ambady (2006), "Math is Hard! The effect of gender priming on women’s attitudes,” Journal of Experimental Social Psychology, 42, pp.428-436.

Svenson, Ola (1981), “Are we all less risky and more skillful than our fellow drivers?” Acta Psychologica, 47(2), pp.143-148. 
Table 1. Gender difference in performance

\begin{tabular}{|c|c|c|c|c|c|c|}
\hline & \multicolumn{3}{|c|}{$\underline{\text { Task } 1 \text { (piece rate) }}$} & \multicolumn{3}{|c|}{ Task 2 (tournament) } \\
\hline & Obs. & Mean & S.E. & Obs. & Mean & S.E. \\
\hline Male & 67 & 12.209 & 0.291 & 67 & 13.134 & 0.240 \\
\hline Female & 64 & 9.719 & 0.280 & 64 & 11.250 & 0.268 \\
\hline All & 131 & 10.992 & 0.229 & 131 & 12.214 & 0.197 \\
\hline $\begin{array}{l}\text { Gender } \\
\text { Difference }\end{array}$ & & $2.490 * * *$ & 0.405 & & $1.884 * * *$ & 0.359 \\
\hline
\end{tabular}

Notes: $* * *$ measure significance at $1 \%$. Because of system-related issues, one observation is missing. 
Table 2. Change in performance (from Task 1 to Task 2)

\begin{tabular}{lcccccc}
\hline & \multicolumn{3}{c}{ Increase in performance } & \multicolumn{3}{c}{ Rate of increase } \\
& Obs. & Mean & S.E. & Obs. & Mean & S.E. \\
\hline Male & 67 & $0.925^{* * *}$ & 0.203 & 67 & $0.094^{* * *}$ & 0.018 \\
Female & 64 & $1.531^{* * *}$ & 0.208 & 64 & $0.185^{* * *}$ & 0.027 \\
All & 131 & $1.221^{* * *}$ & 0.147 & 131 & $0.139^{* * *}$ & 0.017 \\
\hline Gender & & & & & & \\
Difference & & $-0.606^{* *}$ & 0.291 & & $-0.091^{* * *}$ & 0.032 \\
\hline \hline
\end{tabular}

Notes: ${ }^{* *}$ and $* * *$ measure significance at $5 \%$ and $1 \%$ confidence levels. Because of system-related issues, one observation is missing. 
Table 3. Performance by expected rankings on Task 1

\begin{tabular}{lccccc}
\hline \hline $\begin{array}{l}\text { Expected ranking } \\
\text { on task 1 }\end{array}$ & Obs. & $\begin{array}{c}\text { Task } 1 \\
\text { (piece rate) }\end{array}$ & $\begin{array}{c}\text { Task } 2 \\
\text { (tournament) }\end{array}$ & $\begin{array}{c}\text { Increase in } \\
\text { performance }\end{array}$ & Rate of \\
\hline First & 28 & 12.714 & 13.321 & $0.607^{* *}$ & $0.060^{* *}$ \\
& & $(2.386)$ & $(2.161)$ & $(1.524)$ & $(0.127)$ \\
Second & 10.500 & 11.984 & $1.484^{* * *}$ & $0.167^{* * *}$ \\
& & $(2.455)$ & $(2.229)$ & $(1.667)$ & $(0.192)$ \\
Third & 38 & 10.711 & 11.842 & $1.132^{* * *}$ & $0.130^{* * *}$ \\
& & $(2.535)$ & $(2.236)$ & $(1.663)$ & $(0.177)$ \\
Fourth & 8.667 & 11.333 & $2.667 *$ & 0.403 \\
\hline \hline
\end{tabular}

Notes: The table shows subjects' performance by expected rankings on Task 1. *, **, and $* * *$ measure significance at $10 \%, 5 \%$, and $1 \%$, respectively. Because of system-related issues, one observation is missing. 
Table 4. Gender differences in overconfidence

\begin{tabular}{lccccc}
\hline \hline & \multicolumn{2}{c}{ Overconfidence 1 } & & \multicolumn{2}{c}{ Overconfidence 2 } \\
& Obs. & Mean & Obs. & Mean \\
\hline Male & 66 & 0.043 & 66 & $0.265^{* *}$ \\
Female & 62 & $0.502^{* * *}$ & 62 & $0.524^{* * *}$ \\
All & 128 & $0.266^{* * *}$ & 128 & $0.391^{* * *}$ \\
\hline Gender Difference & \multicolumn{3}{c}{$-0.459^{* * *}$} & & $-0.259^{*}$ \\
\hline \hline
\end{tabular}

Notes: The table shows means of two overconfidence measures and gender differences between them. In both measures, a positive (negative) sign implies that subjects are overconfident (under-confident). *, **, and *** measure significance at $10 \%, 5 \%$, and $1 \%$, respectively. Because of computer trouble, one observation is missing. In addition to it, we exclude 3 observations that belong to the same group with her because our method requires sample size by a factor of four when we calculate overconfidence measures. 
Table 5. Gender and performance

\begin{tabular}{lcccc}
\hline \hline & Gender & Obs. & $\begin{array}{c}\text { Increase in } \\
\text { performance }\end{array}$ & Rate of \\
& & & $0.694^{* * *}$ & $0.071^{* * *}$ \\
& 4 males & 36 & $(1.527)$ & $(0.138)$ \\
Male & 2 males and & & $0.923^{* * *}$ & $0.098^{* * *}$ \\
& 2 females & & $(1.742)$ & $(0.160)$ \\
\hline & 4 females & 32 & $1.500^{* * *}$ & $0.174 * * *$ \\
& & & $(1.666)$ & $(0.185)$ \\
Female & 2 males and & & $1.400^{* * *}$ & $0.177^{* * *}$ \\
& 2 females & & $(1.708)$ & $(0.229)$ \\
\hline \hline
\end{tabular}

Notes: ${ }^{* * *}$ measure significance at $1 \%$. Because of computer trouble, one observation is missing. 
Table 6. Gender and overconfidence

\begin{tabular}{|c|c|c|c|c|}
\hline & Gender & Obs. & Overconfidence 1 & Overconfidence 2 \\
\hline \multirow{4}{*}{ Male } & \multirow[b]{2}{*}{4 male } & \multirow[b]{2}{*}{36} & -0.027 & 0.226 \\
\hline & & & & (1.007) \\
\hline & 2 males and & \multirow{2}{*}{24} & 0.043 & 0.235 \\
\hline & & & $(0.764)$ & $(0.894)$ \\
\hline \multirow{6}{*}{ Female } & \multirow{3}{*}{4 female } & \multirow{3}{*}{32} & $0.606 * * *$ & $0.731 * * *$ \\
\hline & & & & \\
\hline & & & $(0.726)$ & $(0.933)$ \\
\hline & \multirow{2}{*}{2 males and } & & & \\
\hline & & \multirow{2}{*}{24} & $0.437 * *$ & $0.371^{*}$ \\
\hline & 2 females & & $(0.965)$ & $(0.981)$ \\
\hline
\end{tabular}

Notes: The table shows means of two overconfidence measures by gender and gender. In both measures, a positive (negative) sign means that people are overconfident (under-confident). *, **, and *** measure significance at $10 \%, 5 \%$, and $1 \%$, respectively. Because of system-related issues, one observation is missing. In addition to it, we exclude 3 observations that belong to the same group with her because our method requires sample size by a factor of four when we calculate overconfidence measures. 
Table 7. Effects of expected rankings and overconfidence on increase in performance

\begin{tabular}{|c|c|c|c|c|}
\hline & Eq.1 & Eq.2 & Eq.3 & Eq.4 \\
\hline \multirow{2}{*}{ Constant } & $0.925^{* * *}$ & $0.992 * * *$ & $0.552 *$ & 0.449 \\
\hline & $(0.203)$ & $(0.187)$ & $(0.315)$ & $(0.288)$ \\
\hline \multirow{2}{*}{ Female dummy } & $0.606 * *$ & 0.120 & 0.385 & -0.270 \\
\hline & $(0.291)$ & $(0.279)$ & $(0.312)$ & $(0.300)$ \\
\hline \multirow{2}{*}{ Overconfidence 1} & & $0.843^{* * *}$ & & $0.974 * * *$ \\
\hline & & $(0.166)$ & & $(0.173)$ \\
\hline \multirow{2}{*}{ Second } & & & $0.715^{*}$ & $0.742 * *$ \\
\hline & & & $(0.398)$ & $(0.359)$ \\
\hline \multirow{2}{*}{ Third } & & & 0.357 & $1.015^{* *}$ \\
\hline & & & $(0.433)$ & $(0.411)$ \\
\hline \multirow[b]{2}{*}{ Fourth } & & & $1.730^{*}$ & $2.447 * *$ \\
\hline & & & (1.037) & $(0.936)$ \\
\hline Obs. & 131 & 128 & 131 & 128 \\
\hline $\mathrm{R}^{2}$ & 0.033 & 0.193 & 0.069 & 0.254 \\
\hline
\end{tabular}

Notes: The table shows the estimation results by OLS regressions. The dependent variable is increase in performance from Task 1 (piece rate) to Task 2 (tournament). *, $* *$, and *** measure significance at $10 \%, 5 \%$, and $1 \%$, respectively. Because of system-related issues, one observation is missing. In addition to it, in estimating Eq.2 and Eq.4, we exclude 3 observations that belong to the same group with her because our method requires sample size by a factor of four when we calculate overconfidence measures. 
Table 8. Effects of expected rankings and overconfidence on rate of increase in performance

\begin{tabular}{lllll}
\hline \hline & Eq.1 & Eq.2 & Eq.3 & Eq.4 \\
\hline Constant & $0.094^{* * *}$ & $0.099^{* * *}$ & 0.051 & 0.030 \\
& $(0.022)$ & $(0.020)$ & $(0.034)$ & $(0.030)$ \\
Female dummy & $0.091^{* * *}$ & 0.031 & $0.061^{*}$ & -0.024 \\
& $(0.032)$ & $(0.030)$ & $(0.034)$ & $(0.031)$ \\
Overconfidence 1 & & $0.112^{* * *}$ & & $0.132^{* * *}$ \\
& & $(0.018)$ & & $(0.018)$ \\
Second & & & $0.082^{*}$ & $0.091^{* *}$ \\
& & & $(0.044)$ & $(0.037)$ \\
Third & & & 0.043 & $0.136^{* * *}$ \\
Fourth & & & $(0.047)$ & $(0.042)$ \\
\hline \hline
\end{tabular}

Notes: The table shows the result by OLS regressions. The dependent variable is the rate of increase in performance from Task 1 (piece rate) to Task 2 (tournament). *, **, and $* * *$ measure significance at $10 \%, 5 \%$, and $1 \%$, respectively. Because of system-related issues, one observation is missing. In addition to it, in estimating Eq.2 and Eq.4, we exclude 3 observations that belong to the same group with her because our method requires sample size by a factor of four when we calculate overconfidence measures. 
Table 9. Effects of expected rankings and overconfidence on increase in performance from Task 2 (tournament) to Task 3 (tournament)

\begin{tabular}{lcccc}
\hline \hline & \multicolumn{2}{c}{ Increase in performance } & \multicolumn{2}{c}{ Rate of increase } \\
& Eq.1 & Eq.2 & Eq.3 & Eq.4 \\
\hline Constant & 0.122 & -0.625 & 0.014 & -0.062 \\
& $(0.296)$ & $(0.414)$ & $(0.029)$ & $(0.041)$ \\
Female dummy & 0.489 & 0.169 & $0.089^{*}$ & 0.060 \\
& $(0.536)$ & $(0.576)$ & $(0.053)$ & $(0.057)$ \\
Overconfidence 1 & & $0.802^{* * * *}$ & & $0.086^{* * *}$ \\
& & $(0.294)$ & & $(0.029)$ \\
Second & & $1.085^{* *}$ & & $0.113^{* *}$ \\
\hline \hline
\end{tabular}

Notes: The table shows the result by OLS regressions. The dependent variables are increases in performance from Task 2 to Task 3 in Eq.1 and Eq.2 and the rate of increase in performance from Task 2 to Task 3 in Eq.3 and Eq.4. ** and *** measure significance at $5 \%$ and $1 \%$. 
Table 10. Effects of expected rankings and overconfidence on increase in performance by subjects’ maze-solving skills

\begin{tabular}{|c|c|c|c|c|}
\hline & \multicolumn{2}{|c|}{$\underline{\text { Increase in performance }}$} & \multicolumn{2}{|c|}{$\underline{\text { Rate of increase }}$} \\
\hline & Bottom half & Upper half & Bottom half & Upper half \\
\hline \multirow{2}{*}{ Constant } & -0.102 & -0.838 & -0.009 & -0.073 \\
\hline & $(1.344)$ & $(1.027)$ & $(0.169)$ & $(0.084)$ \\
\hline \multirow[b]{2}{*}{ Female dummy } & $-0.971^{* *}$ & 0.053 & -0.070 & 0.011 \\
\hline & $(0.426)$ & $(0.481)$ & $(0.053)$ & $(0.039)$ \\
\hline \multirow{2}{*}{ Overconfidence 1} & 0.357 & $2.019 * * *$ & $0.097 * *$ & $0.160 * * *$ \\
\hline & $(0.292)$ & $(0.497)$ & $(0.037)$ & $(0.041)$ \\
\hline \multirow{2}{*}{ Second } & $1.280^{*}$ & $1.038 * *$ & $0.160^{*}$ & $0.088 * *$ \\
\hline & $(0.681)$ & $(0.516)$ & $(0.085)$ & $(0.042)$ \\
\hline \multirow{2}{*}{ Third } & 1.252 & $1.576^{* *}$ & $0.177 *$ & $0.126^{* *}$ \\
\hline & $(0.777)$ & $(0.679)$ & $(0.098)$ & $(0.056)$ \\
\hline \multirow{2}{*}{ Fourth } & $3.577 * * *$ & $3.211^{*}$ & $0.579 * * *$ & 0.249 \\
\hline & (1.209) & $(1.885)$ & $(0.152)$ & $(0.155)$ \\
\hline Observations & 64 & 64 & 64 & 64 \\
\hline $\mathrm{R}^{2}$ & 0.225 & 0.291 & 0.305 & 0.286 \\
\hline
\end{tabular}

Notes: The table presents results from OLS regression. The dependent variables are the increase in performance and rate of increase in performance from Task 1 to Task 2. Standard errors are reported in parentheses. *, **, and *** measure significance at $10 \%$, $5 \%$, and $1 \%$, respectively. "Bottom half” ("Upper half”) refers to subjects whose average ranking in a group of four subjects generated by 1 million times random grouping is above (below) median (2.165). Because of system-related issues, one observation is missing. In addition to it, we exclude 3 observations that belong to the same group with her because our method requires sample size by a factor of four when we calculate overconfidence measures. 


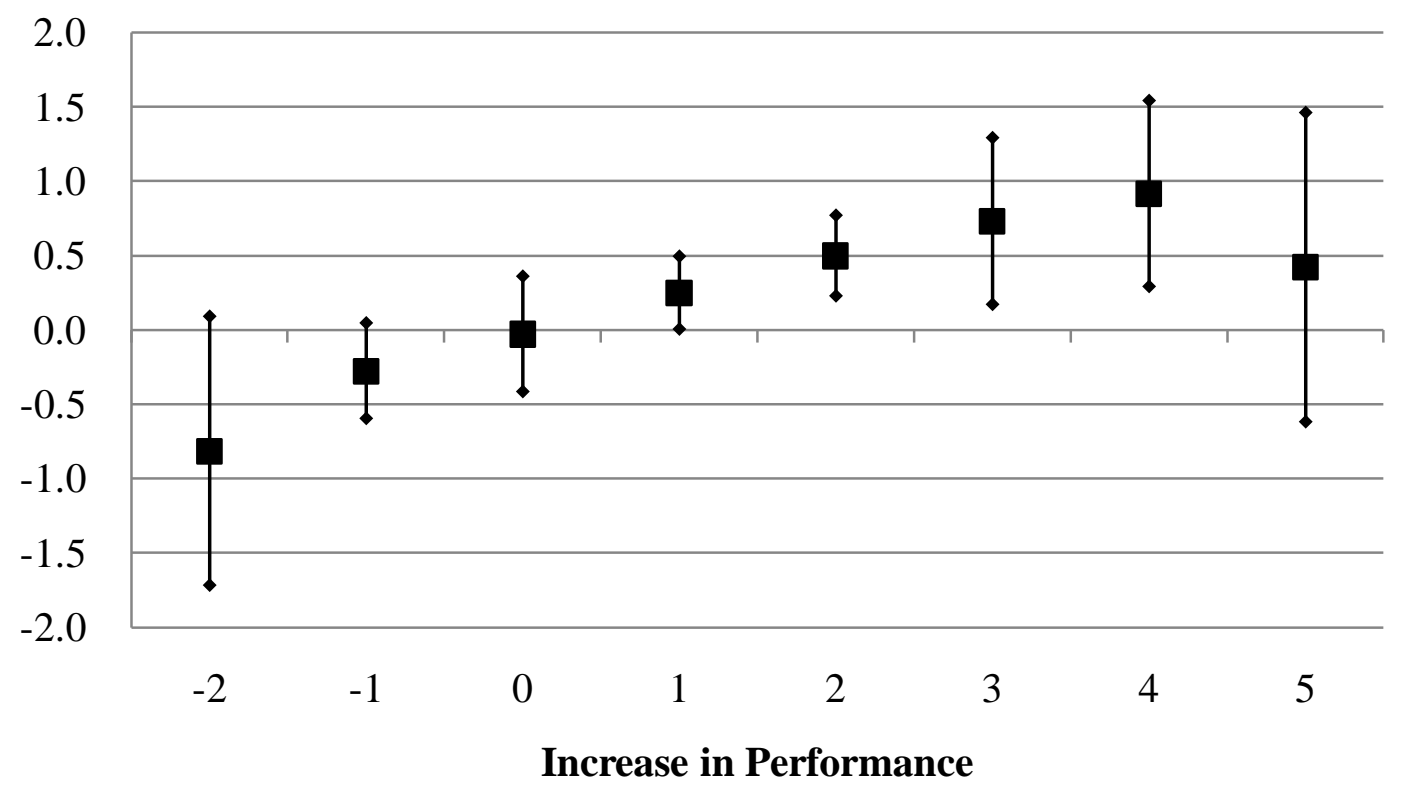

Figure 1. Positive correlation between overconfidence and increase in performance

Notes: Figure 1 shows the arithmetic means and 95\% confidence intervals of Overconfidence 1 by the change in performance from Task 1 to Task 2 . The horizontal and vertical axes represent the change in performance from Task 1 to Task 2 and the degree of overconfidence, respectively. 